\title{
Entanglement in N-harmonium: bosons and fermions
}

\author{
C. L. Benavides-Riveros ${ }^{1,2}$, I. V. Toranzo ${ }^{3}$, and J. S. Dehesa ${ }^{3}$ \\ ${ }^{1}$ Departamento de Física Teórica, \\ Universidad de Zaragoza, 50009 Zaragoza, Spain \\ ${ }^{2}$ Instituto de Biocomputación y Física de Sistemas Complejos, \\ Mariano Esquillor (Edificio I+D), 50018 Zaragoza, Spain \\ ${ }^{3}$ Instituto Carlos I de Física Teórica y Computacional \\ and Departamento de Física Atómica Molecular y Nuclear, \\ Universidad de Granada, 18071 Granada, Spain.
}

June 14, 2021

\begin{abstract}
The ground-state entanglement of a single particle of the N-harmonium system (i.e., a completely-integrable model of $N$ particles where both the confinement and the two-particle interaction are harmonic) is shown to be analytically determined in terms of $N$ and the relative interaction strength. For bosons, we compute the von Neumann entropy of the one-body reduced density matrix by using the corresponding natural occupation numbers. There exists a critical number $N_{c}$ of particles so that below it, for positive values of the coupling constant, the entanglement grows when the number of particles is increasing; the opposite occurs for $N>N_{c}$. For fermions, we compute the one-body reduced density matrix for the closed-shell spinned case. In the strong coupling regime, the linear entropy of the system decreases when $N$ is growing. For fixed $N$, the entanglement is found (a) to decrease (increase) for negatively (positively) increasing values of the coupling constant, and (b) to grow when the energy is increasing. Moreover, the spatial and spin contributions to the total entanglement are found to be of comparable size.
\end{abstract}

PACS numbers: 31.15.-p, 03.67.-a, 05.30.Fk 


\section{Introduction}

The most precise determination of the properties of finite many-electron systems is usually done by means of a full-configuration-interaction (FCI) method, where the solution of the corresponding Schrödinger equation in a given one-electron basis is expressed in terms of a linear combination of all possible Slater determinants. Its application is naturally reduced to a bunch of small systems because of the enormous number of the involved determinants $[1,2]$.

Let us highlight that for all FCI approaches the correlation effects, which remain solely in the wave function, are not described by any correlation operator. Moreover, these effects are usually numerically computed as a difference of two variational energy bounds. Thus, up to now the electron correlation is widely and implicitly believed to be a purely methodical effect coming from the inadequate use of a trial wave function of multiconfiguration Hartree-Fock type; so, lacking of physical reality.

The application of quantum information ideas and techniques in electronic structure theory has recently allowed to conclude that the electron correlation is closely related to entanglement of electrons. Indeed, it has been proved that while the single Slater determinant in the monoconfigurational Hartree-Fock approximation is a disentangled state, the wavefunction of the multiconfiguration Hartree-Fock approximations (such as, e.g. FCI) accounts for entanglement effects. Therefore, entanglement plays an essential role not only in quantum communication between parties separated by macroscopic distances (see e.g., [3,4]), but also it is essential to characterize quantum correlations at short distances. The latter problem, where one should necessarily consider the indistinguishable character of the involved particles, has received relatively less attention until a short time ago [5-10]. This is a serious lack because of its relevance for quantum information processing in various physical systems (see e.g., $[5,11])$, to gain deeper insight into non-classical correlations of atomic and molecular systems as well as to fully understand the course of their dissociation processes and chemical reactions $[10-12]$.

The main difficulty, however, stems from the fact that the Schrödinger equation of most quantum many-body systems cannot be solved analytically. Even numerically, the determination of the wavefunction is, in general, a serious problem. In the last three years an intense effort has been made to determine the entanglement of some real atomic and molecular species such as helium-like atoms [13-17], of a few processes of diatomic molecules [18] and elementary chemical reactions [19]. These works basically focus on the entanglement of bipartite systems, mainly because the characterization of this phenomenon for systems of many indistinguishable constituents is much less known, even at the level of the very notion of entanglement measure [20].

Thus, the quantification of entanglement of bound states for model systems enabling analytic solutions of the associated Schrödinger equation is being a promising way to investigate correlation phenomena. Indeed, entanglement between the constituents of any bound system is most conveniently analyzed in such models, enabling to relate it to the bosonic or 
fermionic character. Up to now, however, only entanglement of some models of two bound electrons have been determined. We refer to the 2-harmonium (or Moshinsky) [21-25], Crandall and Hooke [13] atoms. In all these models the electron confinement is harmonic, and the electron-electron interaction is of harmonic (2-harmonium), $r_{12}^{-1}$ (Crandall), and Coulombic (Hooke) type. All of them show that when the spin degree of freedom and the indistinguishability of electrons are taken into account, new entanglement aspects [24] are encountered as compared to the model of distinguishable particles, although some further clarification is needed.

It is also worth noting that some entanglement features of the 2-harmonium atom are also qualitatively reproduced by the other two models, which give a good description for certain two-fermion systems (e.g., two electrons in a quantum dot is approximately described by the Hooke model). Namely, the growth of the entanglement when either the relative strength or the excitation energy is increasing. It is most interesting that recently the entanglement of some real helium-like atoms has been numerically shown to have an increasing dependence on the energy. This has been done both by the use of very accurate one-electron basis functions of Hylleraas-Kinoshita type $[14,17]$ and some Gaussian or Slater type orbital basis sets $[6,15]$. As well it is observed from the models that the entanglement decrease of these systems in terms of the nuclear charge $\mathrm{Z}$ can also be understood as the result of the relative decrease of the electron-electron-interaction.

In this work we will study the entanglement of the one-body reduced density matrix of the N-harmonium model for bosons and fermions analytically. Von Neumann (for bosons) and linear (for fermions) entropies will be used. The one-body reduced matrix for the spinless fermionic case has been previously computed [26]. We obtain for the first time an explicit expression of the one-body reduced density matrix for the closed-shell spinned fermioniccase. This study will allow us to show that some entanglement features of finite many-particle systems can be understood by purely kinematical considerations to a certain extent. The N-particle harmonium is a completely-integrable system with an arbitrary number $N$ of particles where both the confinement and the two-particle interaction are harmonic. This model has also been used to study cold atoms [27] and to gain further insight into numerous phenomena of a variety of physical systems up to black holes (see e.g., [21,28-34]).

The paper is structured as follows. First, in Section 2 we briefly formulate the quantummechanical problem of the N-particle harmonium, showing its separability by using the appropriate set of normal coordinates [35], and fixing our notational settings. Then, in Section 3 we discuss the general mathematical structure of the one-body reduced density matrix in the bosonic case, obtaining its explicit expression. Moreover, we compute and discuss the analytical expression of the von Neumann entropy of the one-body reduced density matrix for the N-boson harmonium. Further, in Sections 4 and 5 we obtain explicit expressions for the one-body reduced density matrix of the spinned $\mathrm{N}$-fermion harmonium and analyze the linear entropy of the one-body reduced density matrix, with and without the spin degree of freedom. Finally, some concluding remarks and two appendices are given. 


\section{The N-harmonium problem}

The N-harmonium model is a system of $N$ interacting particles (fermions or bosons) which interact harmonically in a three-dimensional harmonic well. It is characterized by the Hamiltonian

$$
H=\frac{1}{2} \sum_{i=1}^{N}\left|\boldsymbol{p}_{i}\right|^{2}+\frac{k}{2} \sum_{i=1}^{N}\left|\boldsymbol{r}_{i}\right|^{2}+\frac{\delta}{2} \sum_{i<j}^{N} r_{i j}^{2},
$$

where $r_{i j}:=\left|\boldsymbol{r}_{i}-\boldsymbol{r}_{j}\right|, k$ is the coupling constant of the harmonic well and $\delta$ the coupling of the harmonic interaction between the particles. The treatment of harmonically interacting bosons by means of this Hamiltonian represents the first exact solution of a N-particle system using only conditions on the reduced space of two-particle density matrices [36]. This system can be expressed in a separable form (i.e., as a system of uncoupled oscillators) using the set of normal coordinates $\left\{\boldsymbol{\xi}_{1}, \ldots, \boldsymbol{\xi}_{N}\right\}$ given by

$$
\boldsymbol{\xi}_{N}:=\frac{1}{\sqrt{N}} \sum_{i=1}^{N} \boldsymbol{r}_{i} \quad \text { and } \quad \boldsymbol{\xi}_{m}:=\frac{1}{\sqrt{m(m+1)}} \sum_{i=1}^{m}\left(\boldsymbol{r}_{i}-\boldsymbol{r}_{m+1}\right)
$$

with $m \in\{1, \ldots, N-1\}$. This is an orthogonal transformation of the position variables. A similar change of coordinates for momenta results in a canonical transformation, preserving the symplectic form. Let us call the new set of momenta $\left\{\boldsymbol{\Xi}_{m}\right\}$. A direct calculation shows that

$$
\sum_{m=1}^{N-1} \boldsymbol{\xi}_{m}^{2}=\frac{1}{N} \sum_{i<j}^{N} r_{i j}^{2}=\sum_{m=1}^{N} \boldsymbol{r}_{m}^{2}-\boldsymbol{\xi}_{N}^{2}
$$

so that the Hamiltonian (1) can be expressed in the following separable form

$$
H=\mathcal{H}_{N}+\sum_{m=1}^{N-1} \mathcal{H}_{m}, \quad \text { where } \quad \mathcal{H}_{N}=\frac{1}{2} \boldsymbol{\Xi}_{N}^{2}+\frac{1}{2} \omega^{2} \boldsymbol{\xi}_{N}^{2} \quad \text { and } \quad \mathcal{H}_{m}=\frac{1}{2} \boldsymbol{\Xi}_{m}^{2}+\frac{1}{2} \mu^{2} \boldsymbol{\xi}_{m}^{2}
$$

where $\omega^{2}:=k$, and $\mu^{2}:=k+N \delta$ depending on the number of particles. Then, the physical solutions of the associated Schrödinger equation (i.e., the wave functions $\Psi\left(\boldsymbol{x}_{1}, \cdots, \boldsymbol{x}_{N}\right)$ ), can be readily obtained. Here, let us first note that (a) there is a ground state whenever $\mu^{2}>0$, and (b) the particles are no longer bound if the relative interaction strength

$$
\frac{\delta}{k} \leq-\frac{1}{N}
$$

On the other hand, let us comment here that the entanglement problem is formulated in terms of reduced density matrices. Either directly from the resulting one-particle reduced 
density matrix or, since the particles are assumed to interact pairwise, in terms of the twoparticle density matrix

$$
\rho\left(\boldsymbol{x}_{1}, \boldsymbol{x}_{2} ; \boldsymbol{y}_{1}, \boldsymbol{y}_{2}\right):=\int d \boldsymbol{x}_{3} \cdots d \boldsymbol{x}_{N}\left|\Psi\left(\boldsymbol{x}_{1}, \boldsymbol{x}_{2}, \boldsymbol{x}_{3}, \cdots, \boldsymbol{x}_{N}\right)\right\rangle\left\langle\Psi\left(\boldsymbol{y}_{1}, \boldsymbol{y}_{2},, \boldsymbol{x}_{3}, \cdots, \boldsymbol{x}_{N}\right)\right|,
$$

which carries all the necessary information required for calculating the quantum-mechanical properties of the whole system. The symbol $\boldsymbol{x}$ stands for spatial and spin coordinates, $\boldsymbol{x}:=(\boldsymbol{r}, \varsigma)$. In particular, the ground state energy of the system can be computed by minimizing a simple linear functional of $\rho$. In passing, let us mention that the N-representability problem for this matrix has proved to be a major challenge for quantum chemistry [37]. The one-body density matrix, which is the basic variable in reduced density matrix functional theory [38], is then given as

$$
\rho_{1}(\boldsymbol{x} ; \boldsymbol{y}):=\int \rho\left(\boldsymbol{x}, \boldsymbol{x}_{2} ; \boldsymbol{y}, \boldsymbol{x}_{2}\right) d \boldsymbol{x}_{2}
$$

By means of the spectral theorem, $\rho_{1}(\boldsymbol{x} ; \boldsymbol{y})$ can be decomposed in terms of its natural spin orbitals $\left\{\phi_{i}(\boldsymbol{x})\right\}$ and its eigenvalues $\left\{n_{i}\right\}$, the natural occupation numbers: $\rho_{1}(\boldsymbol{x} ; \boldsymbol{y})=$ $\sum_{i} n_{i} \phi_{i}(\boldsymbol{x}) \phi_{i}(\boldsymbol{y})$, where $\sum_{i} n_{i}=1$, with (for fermions) $0 \leq n_{i} \leq 1 / N$.

For the case of an assembly of bosons we will compute the von Neumann entropy of the one-body reduced density matrix finding explicitly the occupation numbers of the system, as described in the next section. Recently, there has been a renewed interest in formulating the reduced density matrix theory using Wigner quasidistributions [25, 39, 40]. Our treatment for bosons will be done by using the Wigner function in phase space, what is a natural procedure for these systems. Let us advance that there exists a critical value for the number of particles. Below this critical value (around 3.5), we will show that for positive values of the coupling constant $\delta$ the first occupation number decreases (and consequently the von Neumann entropy grows) as the number of particles is increasing. Above this value, the first occupation number increases as the number of particles is increasing. Moreover, the first occupation number tends to 1 (and the entropy tends to 0) in the limit when the number of particles tends to infinity.

Later on, for fermions we use the one-body reduced density matrix to compute an explicit expression for its purity and its linear entropy as well. Let us advance that we will show that in the region of negative coupling $\delta$, the entropy grows when the number of particles is increasing. A similar situation is observed in the attractive case for small values of the coupling constant. For large values of $\delta / k$ the situation is the opposite: when particles are added to the system the entropy decreases. In both bosonic and fermionic cases, we will show that it is possible to calculate these two measures of entanglement as a function of the coupling constant $\delta / k$ and the number of particles. 


\section{The N-boson harmonium: von Neumann entropy}

In this section we determine the von Neumann entropy of the N-boson harmonium ground state in terms of $N$ and the relative interaction strength $\delta / k$. Taking into account the Gaussian character of the ground-state oscillator wave function, the ground state distribution on phase space is characterized by the Wigner N-body density function

$$
\begin{aligned}
& W_{\mathrm{gs}}^{b}\left(\boldsymbol{r}_{1}, \ldots, \boldsymbol{r}_{N} ; \boldsymbol{p}_{1}, \ldots, \boldsymbol{p}_{N}\right)=\frac{1}{\pi^{3 N}} e^{-2 \mathcal{H}_{N} / \omega} e^{-2 \sum_{m=1}^{N-1} \mathcal{H}_{m} / \mu} \\
& \quad=\frac{1}{\pi^{3 N}} \exp \left[-(\omega-\mu) \boldsymbol{\xi}_{N}^{2}-\mu \sum_{i=1}^{N} \boldsymbol{r}_{i}^{2}+\frac{\omega-\mu}{\omega \mu} \Xi_{N}^{2}-\frac{1}{\mu} \sum_{i=1}^{N} \boldsymbol{p}_{i}^{2}\right] .
\end{aligned}
$$

The energy of the ground state is the total sum of the contributions of each individual oscillator, i.e. $E_{\mathrm{gs}}^{b}=\frac{3}{2}[\omega+(N-1) \mu]$. On the other hand the one-body density factorizes as a product of two separable quantities, to wit:

$$
\begin{aligned}
d_{1}^{b}(\boldsymbol{r} ; \boldsymbol{p}) & :=\int W_{\mathrm{gs}}^{b}\left(\boldsymbol{r}, \boldsymbol{r}_{2}, \ldots, \boldsymbol{r}_{N} ; \boldsymbol{p}, \boldsymbol{p}_{2}, \ldots, \boldsymbol{p}_{N}\right) \prod_{m=2}^{N} d \boldsymbol{r}_{m} d \boldsymbol{p}_{m} \\
& =e^{-\mu \boldsymbol{r}^{2}-\frac{1}{\mu} \boldsymbol{p}^{2}} \Delta^{\boldsymbol{r}}(\boldsymbol{r}, \mu, \omega, N) \Delta^{\boldsymbol{p}}(\boldsymbol{p}, \mu, \omega, N)
\end{aligned}
$$

It can be shown that the $\Delta$-function fulfills the following recursion relation:

$$
\begin{aligned}
\Delta^{r}(\boldsymbol{r}, \mu, \omega, N) & :=\frac{1}{\pi^{3 N / 2}} \int \exp \left[-\frac{\omega-\mu}{N}\left(\boldsymbol{r}+\sum_{m=2}^{N} \boldsymbol{r}_{m}\right)^{2}-\mu \sum_{m=2}^{N} \boldsymbol{r}_{m}^{2}\right] \prod_{m=2}^{N} d \boldsymbol{r}_{m} \\
& =\frac{1}{\mu_{1}^{3 / 2}} \frac{1}{\pi^{3(N-1) / 2}} \int \exp \left[-\frac{\omega-\mu}{N} \frac{\mu}{\mu_{1}}\left(\boldsymbol{r}+\sum_{m=2}^{N-1} \boldsymbol{r}_{m}\right)^{2}-\mu \sum_{m=2}^{N-1} \boldsymbol{r}_{m}^{2}\right] \prod_{m=2}^{N-1} d \boldsymbol{r}_{m} \\
& =\frac{1}{\mu_{1}^{3 / 2}} \frac{1}{\pi^{3(N-1) / 2}} \int \exp \left[-\frac{\omega-\mu_{1}}{N-1} \frac{\mu}{\mu_{1}}\left(\boldsymbol{r}+\sum_{m=2}^{N-1} \boldsymbol{r}_{m}\right)^{2}-\mu \sum_{m=2}^{N-1} \boldsymbol{r}_{m}^{2}\right] \prod_{m=2}^{N-1} d \boldsymbol{r}_{m} \\
& =(\text { const. }) \Delta^{r}\left(\sqrt{\frac{\mu}{\mu_{1}}} \boldsymbol{r}, \mu_{1}, \omega, N-1\right),
\end{aligned}
$$

where $\mu_{m}:=\frac{m}{N} \omega+\frac{N-m}{N} \mu$. The constant (const.) will be determined by normalization so that it will depend on the number of particles as well as on the frequencies. Therefore,

$$
\begin{aligned}
\Delta^{r}(\boldsymbol{r}, \mu, \omega, N) & =(\text { const. }) \Delta^{r}\left(\sqrt{\frac{\mu}{\mu_{1}}} \boldsymbol{r}, \mu_{1}, \omega, N-1\right)=(\text { const. }) \Delta^{r}\left(\sqrt{\frac{\mu}{\mu_{2}}} \boldsymbol{r}, \mu_{2}, \omega, N-2\right) \\
& =\ldots=\text { (const.) } \exp \left(-\frac{\omega-\mu}{N} \frac{\mu}{\mu_{N-1}} \boldsymbol{r}^{2}\right)
\end{aligned}
$$


and in addition that

$$
\Delta^{p}(\boldsymbol{p}, \mu, \omega, N) \equiv \Delta^{\boldsymbol{r}}\left(\boldsymbol{p}, \mu^{-1}, \omega^{-1}, N\right),
$$

so that the expression (9) can be transformed as:

$$
d_{1}^{b}(\boldsymbol{r} ; \boldsymbol{p})=\frac{N^{3}}{\pi^{3}}\left(\frac{\omega \mu}{\mathcal{A}_{N}}\right)^{3 / 2} \exp \left(-\frac{N \omega \mu}{(N-1) \omega+\mu} \boldsymbol{r}^{2}-\frac{N}{\omega+(N-1) \mu} \boldsymbol{p}^{2}\right),
$$

where $\mathcal{A}_{N}=[(N-1) \omega+\mu][\omega+(N-1) \mu]$.

Let us now diagonalize this expression by decomposing it into its set of natural orbitals as well as into its occupation numbers. The task is made easier by noting that the one-body quasidensity is a linear combination of Laguerre polynomials (the phase-space counterpart of the Hermite polynomials). To see that, let us define

$$
\gamma_{N}:=(\omega \mu)^{1 / 4}\left[\frac{\omega+(N-1) \mu}{(N-1) \omega+\mu}\right]^{1 / 4}, \quad \lambda_{N}:=\frac{N \sqrt{\omega \mu}}{\sqrt{\mathcal{A}_{N}}},
$$

as well as the symplectic transformation

$$
S_{N}:=\left(\begin{array}{cc}
\gamma_{N} & 0 \\
0 & \gamma_{N}^{-1}
\end{array}\right) \quad \text { and } \quad U:=S_{N} \boldsymbol{u} \quad \text { where } \quad \boldsymbol{u}^{T}=(\boldsymbol{r}, \boldsymbol{p}) .
$$

Then the one-body quasidensity function can be rewritten in the Gaussian way as follows,

$$
d_{1}^{b}(U)=\frac{\lambda_{N}^{3}}{\pi^{3}} e^{-\lambda_{N} U^{2}}
$$

showing that it is actually a Gibbs state. Note that $\lambda_{N} \leq 1$ for all $N$, since $\omega+\mu \geq 2 \sqrt{\omega \mu}$ and $\mathcal{A}_{N} \geq N^{2} \omega \mu$ and that $\lambda_{N} \rightarrow 1$ when $N \rightarrow \infty$.

It is known that associated to any symplectic transformation (say, $S_{N}$ ), there is a unitary operator acting on the Hilbert space [41]. Let us use this transformation to find the set of occupation numbers in the basis of Wigner eigenfunctions of the harmonic oscillator. Since the one-body quasidensity function factorizes completely, from now on we work in one dimension. From the series formula

$$
(1-t) \sum_{r=0}^{\infty} L_{r}(x) e^{-x / 2} t^{r}=e^{-\frac{1+t}{1-t} \frac{x}{2}}
$$

where $L_{r}(x)$ is the Laguerre polynomial, it follows that

$$
d_{1}^{b}(U)=\sum_{r=0}^{\infty} \frac{(-1)^{r}}{\pi} L_{r}\left(2 U^{2}\right) e^{-U^{2}} n_{r}
$$


where the occupation numbers are equal to

$$
n_{r}=\frac{2 \lambda_{N}}{1+\lambda_{N}}\left(\frac{1-\lambda_{N}}{1+\lambda_{N}}\right)^{r}=:\left(1-t_{N}\right) t_{N}^{r},
$$

fulfilling $\sum_{r} n_{r}=1$ as one should expect.

For the sake of completeness we plot the numerical behavior of the one-body quasidensity function of the N-boson harmonium in Figure 1 for different values of the number of bosons. We observe that, as the number of bosons grows, the profiles of the position and momentum densities become narrower and wider, respectively. This clearly indicates that the more precisely the particles are localized in position space, the larger the localization in momentum space, as one should expect according to the position-momentum uncertainty principle. There is no relevant difference for negative values of the coupling constant $\delta$ except that in this case it can be plotted only when $\delta / k \geq-1 / N$.

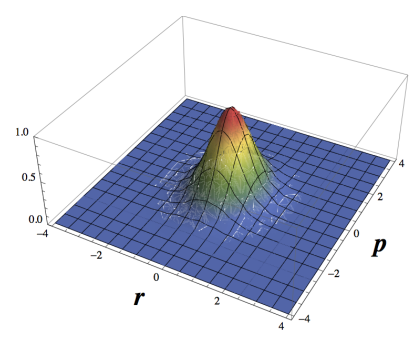

(a) $N=2$

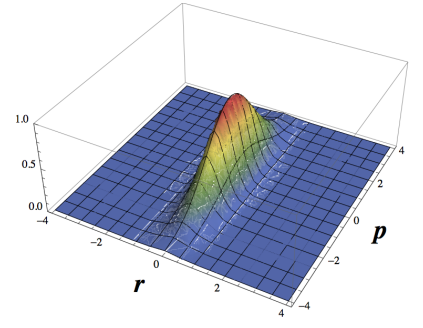

(c) $N=20$

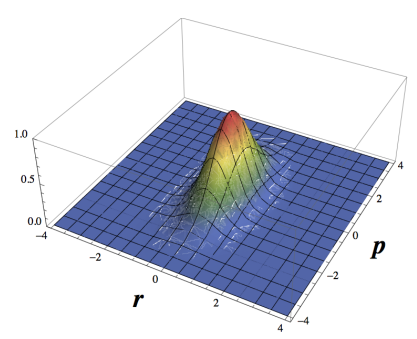

(b) $N=6$

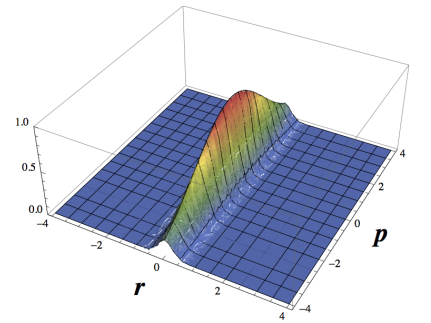

(d) $N=50$

Figure 1: The one-body quasidensity function (13) is plotted for different values of the number of bosons. Since the one-body quasidensity function factorizes completely, we plot it in one spatial dimension (two dimensions in phase space). The strength $\delta / k$ is taken to be equal to 1 . Note that as the number of bosons grows, the profiles of the position and momentum densities become narrower and wider, respectively.

Finally we can compute the von Neumann entropy of the N-boson harmonium explicitly in terms of $N$ and the relative interaction strength $\delta / k$, obtaining the value

$$
\begin{aligned}
S(N) & :=-\sum_{r=0} n_{r} \log n_{r}=-\log \left(1-t_{N}\right)-\frac{t_{N} \log \left(1-t_{N}\right)}{1-t_{N}} \\
& =-\frac{\sqrt{\mathcal{A}_{N}}+N \sqrt{\omega \mu}}{2 N \sqrt{\omega \mu}} \log \left[\frac{2 N \sqrt{\omega \mu}}{\sqrt{\mathcal{A}_{N}}+N \sqrt{\omega \mu}}\right],
\end{aligned}
$$


which complements and extends a similar formula encountered by other means in a black-hole context [30].

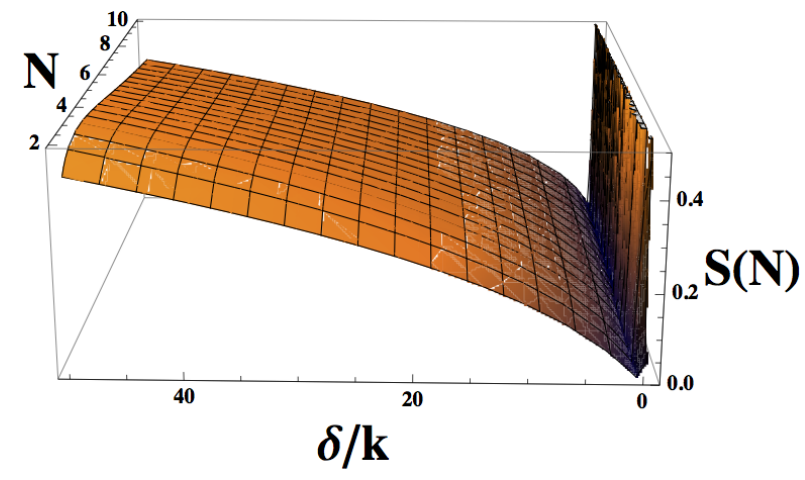

Figure 2: Von Neumann entropy of the one-body reduced density matrix for the N-boson harmonium is plotted as a function of the number of bosons $N$ and the relative interaction strength $\delta / k$. It is apparent that there is a critical point around $N_{c} \sim 3.5$, where the entanglement acquires its maximum value.
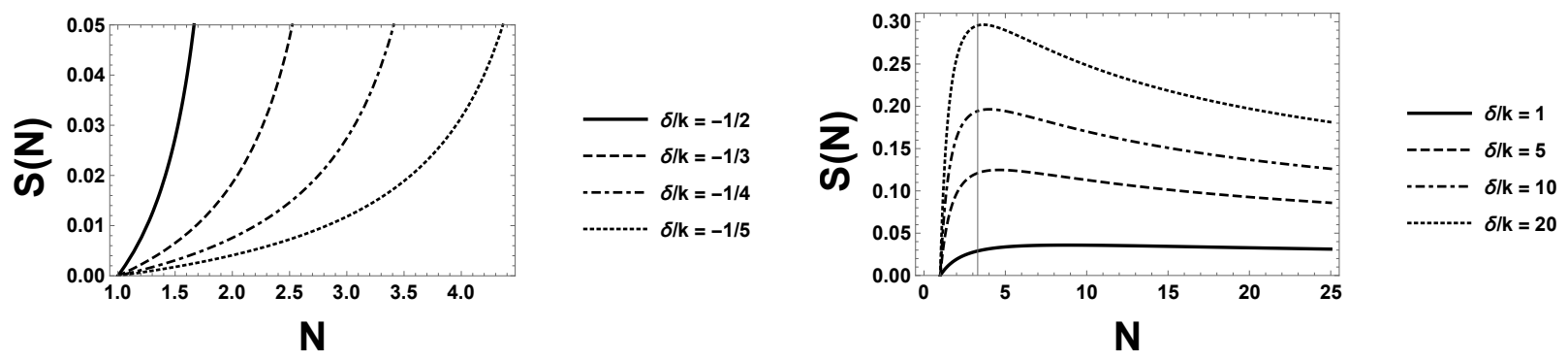

Figure 3: Von Neumann entropy of the one-body reduced density matrix for the N-boson harmonium, whose explicit formula is given in Eq. (20), is plotted as a function of the number of particles for eight different values of the relative interaction strength $\delta / k$. Note that for each value of the relative strength there is a peak around the critical value $N_{c}$.

This entanglement measure is numerically examined in Figure 2 in terms of the number $N$ of bosons and $\delta / k$. As it is stated in (5) the minimum value of this relative strength is $-1 / N$. There is a critical point around $N_{c} \sim 3.5$, where the entanglement acquires its maximum value. This is more clearly seen in Figure 3, where the explicit dependence of the von Neuman entropy on $N$ is shown. To investigate it in a closer way, we study the dependence of the occupation numbers on the number of bosons and the relative interaction strength. In particular, we plot the first occupation number separately in terms of $N$ and the relative strength. This is done in Figure 4. Therein, we note that below a critical value of number of particles (around $N_{c} \sim 3.5$ ), for positive values of the coupling constant $\delta / k$, the first occupation number decreases as the number of particles increases. Beyond this value the situation is reversed: the value of the first occupation number increases as the number of particles increases. This implies that $n_{0} \sim 1$ in the limit when the number of 
particles tends to infinite, which is a necessary condition for the existence of a Bose-Einstein condensation [42].

In summary, the critical point around $N \sim 3.5$ where the von Neumann entropy is maximum is closely connected with the minimum of the first occupation number occurring at such position. It is found that above this critical value the spatial entanglement decreases when we add up more and more particles to the system, so that the degrowth rate increases when the coupling constant is increasing. Moreover, it can be shown that the entropy vanishes when $N$ goes to infinite.

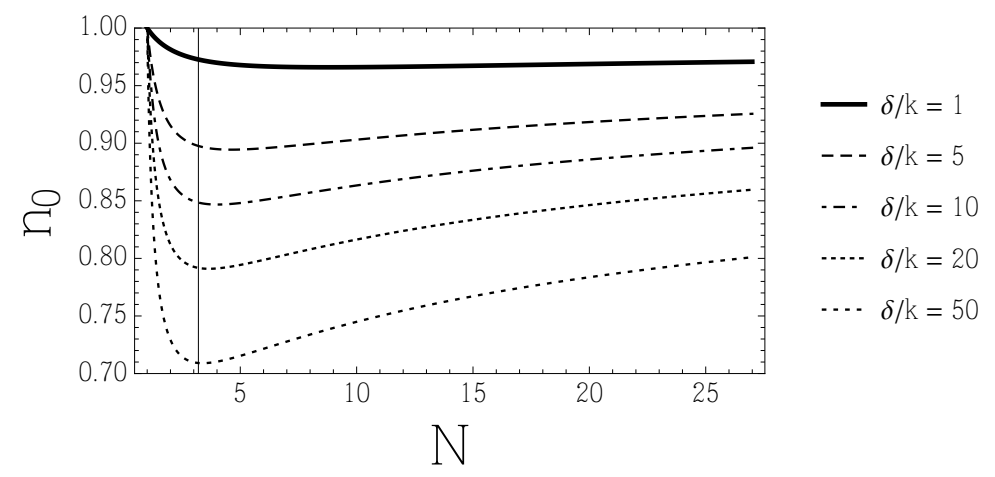

Figure 4: Plot of the first occupation number $n_{0}=1-t_{N}$ as a function of the number of particles for different positive values of $\delta / k$. As it is displayed, $n_{0}$ takes its minimum value around a critical value in the number of particles $(\sim 3.5)$.

\section{The spinless N-fermion harmonium: linear entropy}

In this section we study the spatial entanglement of the N-fermion harmonium (i.e., entanglement without taking into account the spin degree of freedom of the constituents) using the linear entropy of the one-particle density matrix as measure of entanglement of one particle. This measure is a first order approximation of the von Neumann entropy of the reduced one-body density of the system. In fact, it is a lower bound of this entropy. We will consider the case in which the spinless fermions are confined in a one-dimensional well, mainly because the antisymmetric fermion case requires more elaborate computations. Here we have to consider the antisymmetry under exchange of position coordinates $\left\{r_{1}, \ldots, r_{N}\right\}$.

Taking into account the Hamiltonian (1) for the N-fermion harmonium, one has that the ground-state energy of the system is

$$
E_{\mathrm{gs}}^{f}=\frac{1}{2} \omega+\sum_{j=1}^{N-1} \mu\left(m+\frac{1}{2}\right)=\frac{1}{2} \omega+\frac{1}{2} \mu\left(N^{2}-1\right)
$$


and the corresponding eigenfunction can be expressed as

$$
\Psi^{f}\left(\xi_{1}, \ldots, \xi_{N}\right)=\frac{1}{\sqrt{N !}} \sum_{J \in S_{N}}(-)^{J} J\left[\phi_{n_{N}}^{\omega}\left(\xi_{N}\right) \prod_{m=1}^{N-1} \phi_{n_{m}}^{\mu}\left(\xi_{m}\right)\right]
$$

where $n_{m} \in\{0, \cdots, N-1\}$, so that $n_{i} \neq n_{j}$ whenever $i \neq j$. The symbol $J$ denotes an element of the permutation group $S_{N}$ of $N$ elements (acting on the $r$-coordinates), and $\phi_{n}^{\nu}$ is the single-particle wave function given by an Hermite function of degree $n$ with frequency $\nu$. The collective mode $\xi_{N}$ is symmetric under any exchange of the position coordinates. Therefore, one has $n_{N}=0$; otherwise one would have $n_{m}=0$ for some $m \neq N$, and the wave function would not be totally antisymmetric. This eigenfunction can be rewritten as

$$
\Psi_{\mathrm{gs}}^{f}\left(\xi_{1}, \ldots, \xi_{N}\right)=\text { (const.) } \sum_{J \in S_{N}}(-)^{J} J\left[\prod_{m=1}^{N-1} H_{n_{m}}\left(\sqrt{\mu} \xi_{m}\right)\right] e^{-\frac{\omega}{2} \xi_{N}^{2}-\frac{\mu}{2} \sum_{m=1}^{N-1} \xi_{m}^{2}}
$$

where $H_{n}$ is the Hermite polynomial of degree $n$ and now $n_{m} \in\{1, \cdots, N-1\}$. The exponential power of (23) is the bosonic wave function, counterpart of the Wigner function in $(8)$.

As an illustrative case let us consider the 3-harmonium system. To produce an antisymmetric wave function there is only one possibility for the degree of the polynomials, namely $n_{1}=1$ and $n_{2}=2$. Then the ground-state eigenfunction is

$$
\Psi_{\mathrm{gs}}^{f}\left(\xi_{1}, \xi_{2}, \xi_{3}\right)=\text { (const.) } \sum_{J \in S_{3}}(-)^{J} J[\psi(\xi)] e^{-\frac{\omega}{2} \xi_{3}^{2}-\frac{\mu}{2}\left(\xi_{1}^{2}+\xi_{2}^{2}\right)}
$$

where $\psi(\xi):=H_{1}\left(\sqrt{\mu} \xi_{1}\right) H_{2}\left(\sqrt{\mu} \xi_{2}\right)$ and $\xi:=\left(\xi_{1}, \xi_{2}\right)$. Note that the six elements of the permutation group $S_{3}$ are

$$
\begin{aligned}
& p_{1}=\left(\begin{array}{lll}
1 & 2 & 3
\end{array}\right), \quad p_{2}=\left(\begin{array}{lll}
2 & 1 & 3
\end{array}\right), \quad p_{3}=\left(\begin{array}{lll}
2 & 3 & 1
\end{array}\right), \\
& p_{4}=\left(\begin{array}{lll}
1 & 3 & 2
\end{array}\right), \quad p_{5}=\left(\begin{array}{lll}
3 & 1 & 2
\end{array}\right), \quad p_{6}=\left(\begin{array}{lll}
3 & 2 & 1
\end{array}\right) \text {. }
\end{aligned}
$$

The coordinate $\xi$ transforms under the representation of the permutation group in the following form:

$$
\begin{aligned}
& R\left(p_{1}\right) \xi=\xi, \quad R\left(p_{2}\right) \xi=M_{1} \xi, \quad R\left(p_{3}\right) \xi=M_{1} M_{2} \xi \\
& R\left(p_{4}\right) \xi=M_{2} \xi, \quad R\left(p_{5}\right) \xi=M_{1} M_{3} \xi, \quad R\left(p_{6}\right) \xi=M_{3} \xi
\end{aligned}
$$

where

$$
M_{1}=\left(\begin{array}{cc}
-1 & 0 \\
0 & 1
\end{array}\right), \quad M_{2}=\left(\begin{array}{cc}
\cos \pi / 3 & \sin \pi / 3 \\
\sin \pi / 3 & -\cos \pi / 3
\end{array}\right) \quad M_{3}=\left(\begin{array}{cc}
\cos \pi / 3 & -\sin \pi / 3 \\
-\sin \pi / 3 & -\cos \pi / 3
\end{array}\right) .
$$

Note that $\operatorname{det} M_{i}=-1$ and $M^{t} M=1$. Any matrix

$$
M_{\phi}=\left(\begin{array}{cc}
\cos \phi & \sin \phi \\
\sin \phi & -\cos \phi
\end{array}\right)
$$


is a reflection in the axis $\theta=\phi / 2$. In our case, this means $\theta=\pi / 2, \pi / 6,-\pi / 6$; i.e., three reflection axes at 60-degree angles to each other. The rotations $R\left(p_{3}\right)$ and $R\left(p_{5}\right)$ are products of two reflections each. Thus, the eigenfunction transforms as follows:

$$
\begin{aligned}
\Psi_{\mathrm{gs}}^{f}\left(\xi_{1}, \xi_{2}, \xi_{3}\right) & =\text { (const.) } e^{-\frac{\omega}{2} \xi_{3}^{2}-\frac{\mu}{2}\left(\xi_{1}^{2}+\xi_{2}^{2}\right)} \sum_{i=1}^{6}(-)^{i+1} \psi\left(R\left(p_{i}\right) \xi\right) \\
& =\text { (const.) } e^{-\frac{\omega-\mu}{2} \xi_{3}^{2}-\frac{\mu}{2}\left(r_{1}^{2}+r_{2}^{2}+r_{3}^{2}\right)} \sum_{i=1}^{6}(-)^{i+1} \psi\left(R\left(p_{i}\right) \xi\right) \\
& =\text { (const. }) e^{-\frac{\omega-\mu}{2} \xi_{3}^{2}-\frac{\mu}{2}\left(r_{1}^{2}+r_{2}^{2}+r_{3}^{2}\right)}\left(r_{1}-r_{2}\right)\left(r_{1}-r_{3}\right)\left(r_{2}-r_{3}\right) .
\end{aligned}
$$

Notice that the last equality has been possible because

$$
\frac{1}{12 \sqrt{2}} \frac{1}{\mu^{3 / 2}} \sum_{i=1}^{6}(-)^{i+1} \psi\left(\sqrt{\mu} R\left(p_{i}\right) \xi\right)=-\frac{1}{\sqrt{2}}\left(\xi_{1}^{3}-3 \xi_{1} \xi_{2}^{2}\right)=\left(r_{1}-r_{2}\right)\left(r_{1}-r_{3}\right)\left(r_{2}-r_{3}\right)
$$

is a Vandermonde determinant. This 3 -fermion result can be extended to the N-fermion system. In fact, general results of the theory of antisymmetric functions ensure that the wave function of a system of spinless fermions is equal to the wave function of the corresponding bosonic system multiplied by the $N$-variable Vandermonde determinant (see Section 3.1 of [43]). So, the ground-state wave function of the spinless $\mathrm{N}$-fermion harmonium described by the Hamiltonian (1) has the form:

$$
\Psi^{f}\left(r_{1}, \cdots, r_{N}\right)=\text { (const.) } \prod_{i<j}\left(r_{i}-r_{j}\right) \exp \left[-\frac{\omega-\mu}{2} \xi_{N}^{2}-\frac{\mu}{2} \sum_{i=1}^{N} r_{i}^{2}\right],
$$

where the product-like symbol on the right hand of this expression denotes the Vandermonde determinant:

$$
\mathcal{V}_{\left(r_{1}, \cdots, r_{N}\right)}:=\left|\begin{array}{ccc}
1 & \cdots & 1 \\
r_{1} & \cdots & r_{N} \\
\vdots & \ddots & \vdots \\
r_{1}^{N-1} & \cdots & r_{N}^{N-1}
\end{array}\right|=\prod_{1 \leq i<j \leq N}\left(r_{i}-r_{j}\right) .
$$

Concrete calculations $[29,33]$ have borne out this fact several times. On the other hand, it has been pointed out by several authors that this function is actually a generalization of Laughlin's wave function for the fractional quantum Hall effect [44]. This is not surprising since in the Hall effect the magnetic field can be understood as a harmonic potential acting on the electrons. Let us also point out that the Wigner function corresponding to the Gaussian one on the right-hand side of (27) is nothing but the Wigner N-body quasidensity given by (8).

Let us now calculate the one-body density of the one-dimensional spinless $\mathrm{N}$-fermion harmonium, which is defined by

$$
\rho_{1}\left(r ; r^{\prime}\right)=\int d r_{2} \cdots d r_{N} \Psi^{f}\left(r, r_{2}, \cdots, r_{N}\right) \Psi^{f}\left(r^{\prime}, r_{2}, \cdots, r_{N}\right) .
$$


For this purpose it is convenient to rewrite the eigenfunction (27) as:

$$
\Psi^{f}\left(r_{1}, \cdots, r_{N}\right)=(\text { const. }) \mathcal{V}_{\left(r_{1}, \cdots, r_{N}\right)} e^{-a\left(r_{1}^{2}+\cdots+r_{N}^{2}\right)+b_{N}\left(r_{1}+\cdots+r_{N}\right)^{2}},
$$

with $a=\frac{\mu}{2}$ and $b_{N}=\frac{\mu-\omega}{2 N}$. Moreover, we will use the Hubbard-Stratonovich transformation

$$
e^{\alpha \zeta^{2}}=\sqrt{\frac{\alpha}{\pi}} \int_{-\infty}^{\infty} d z e^{-\alpha z^{2}+2 \alpha z \zeta}, \quad \text { with } \quad \alpha \in \mathbb{C} \quad \text { and } \quad \operatorname{Re}(\alpha)>0
$$

where $\zeta=r_{2}+\cdots+r_{N}$ and $\alpha=2 b_{N}$. Then the one-body density reads:

$$
\begin{aligned}
\rho_{1}\left(r ; r^{\prime}\right)=k_{N} & e^{-\left(a-b_{N}\right)\left(r^{2}+r^{\prime 2}\right)} \int d z e^{-2 b_{N} z^{2}} e^{(N-1) \frac{b_{N}^{2}}{2 a}\left(r+r^{\prime}+2 z\right)^{2}} \\
& \times \int d z_{2} \cdots d z_{N} \mathcal{V}_{\left(z_{r}, z_{2}, \cdots, z_{N}\right)} \mathcal{V}_{\left(z_{r}^{\prime}, z_{2}, \cdots, z_{N}\right)} \prod_{j=2}^{N} e^{-z_{j}^{2}}
\end{aligned}
$$

where $k_{N}$ denotes the normalization constant (to be calculated later), and $z_{j}:=\sqrt{2 a}\left[r_{j}-\right.$ $\left.\frac{b_{N}}{2 a}\left(r+r^{\prime}+2 z\right)\right]$ is a convenient change of coordinates. A key observation [26] is that the last expression on the right-hand side is the multiplication of two Slater determinants and therefore, the computation of the reduced density matrix can be done using well-known methods in quantum chemistry. Indeed, with the notation

$$
\begin{gathered}
\beta_{N}:=\sqrt{\frac{\mu-\omega}{(N-1) \omega+\mu}}, \quad q_{\left(r, r^{\prime}\right)}:=\sqrt{\mu}\left[r-\frac{1}{2} \beta_{N}^{2}\left(r+r^{\prime}\right)\right] \\
\quad \text { and } \quad c_{N}:=\frac{(\mu-\omega)^{2}}{(N-1) \omega+\mu} \frac{N-1}{2 N}
\end{gathered}
$$

one can express the one-body density as

$$
\rho_{1}\left(r ; r^{\prime}\right)=k_{N} e^{-a_{N}\left(r^{2}+r^{\prime 2}\right)+2 c_{N} r r^{\prime}} \int d u e^{-u^{2}} \sum_{j=0}^{N-1} \frac{1}{2^{j} j !} H_{j}\left[q_{\left(r, r^{\prime}\right)}-\beta_{N} u\right] H_{j}\left[q_{\left(r^{\prime}, r\right)}-\beta_{N} u\right],
$$

with $a_{N}:=a-b_{N}-c_{N}$. This expression can be calculated analytically with the help of the following general formula derived in Appendix A:

$$
\begin{aligned}
& \int_{-\infty}^{\infty} e^{-u^{2}} H_{k}\left(q_{\left(r, r^{\prime}\right)}-\beta_{N} u\right) H_{k}\left(q_{\left(r^{\prime}, r\right)}-\beta_{N} u\right) d u \\
& =\sum_{\substack{n_{1}, n_{2}=0 \\
n_{1}+n_{2} \text { even }}}^{k}\left(2 \beta_{N}\right)^{n_{1}+n_{2}}\left(\begin{array}{c}
k \\
n_{1}
\end{array}\right)\left(\begin{array}{c}
k \\
n_{2}
\end{array}\right) H_{k-n_{1}}\left(-q_{\left(r, r^{\prime}\right)}\right) H_{k-n_{2}}\left(-q_{\left(r^{\prime}, r\right)}\right) \Gamma\left(\frac{n_{1}+n_{2}+1}{2}\right) .
\end{aligned}
$$

Then the integral at the right-hand side of (34) can be expressed as the following power sum:

$$
\int d u e^{-u^{2}} \sum_{j=0}^{N-1} \frac{1}{2^{j} j !} H_{j}\left[q_{\left(r, r^{\prime}\right)}-\beta_{N} u\right] H_{j}\left[q_{\left(r^{\prime}, r\right)}-\beta_{N} u\right]=\sqrt{\pi} \sum_{t=0}^{N-1} \sum_{s=0}^{2 t} c_{t, s}^{N} r^{2 t-s} r^{\prime s}
$$


where the expansion coefficients $c_{r, s}^{N}$ depend on the frequencies $\omega, \mu$ and the number of particles. When $r+s$ is an odd number or bigger than $2(N-1), c_{r, s}^{N}=0$. By symmetry it is clear that $c_{t, s}^{N}=c_{s, t}^{N}$. In the one fermion case there is only one coefficient $c_{0,0}^{1}=1$. For $N$ fermions, the rank of the quadratic form of the $c_{r, s}$ is $2 N-1$. In particular, when $N=2$, we have the expansion coefficients

$$
c_{0,0}^{2}=\frac{2 \mu}{\omega+\mu}, \quad c_{1,1}^{2}=\frac{\mu\left(\mu^{2}+2 \mu \omega+5 \omega^{2}\right)}{(\omega+\mu)^{2}} \quad \text { and } \quad c_{2,0}^{2}=c_{0,2}^{2}=\frac{\mu(\omega-\mu)(3 \omega+\mu)}{2(\omega+\mu)^{2}} .
$$

For completeness, let us mention that the reader will have no difficulty in verifying that $\rho_{1}(r, r)$ - the diagonal of (29) - coincides (except for the difference in the normalization conventions) with the one obtained in [25, Sect. V] by the Wigner function method. Moreover, for $N=3$ the non-vanishing expansion coefficients are:

$$
\begin{gathered}
c_{0,0}^{3}=\frac{3}{2}\left[1+\frac{(\omega-\mu)^{2}}{(2 \omega+\mu)^{2}}\right], \quad c_{1,1}^{3}=\frac{\mu\left(-15 \omega^{3}+51 \mu \omega^{2}+15 \mu^{2} \omega+3 \mu^{3}\right)}{(2 \omega+\mu)^{3}}, \\
c_{2,2}^{3}=\frac{\mu^{2}\left(363 \omega^{4}+168 \mu \omega^{3}+90 \mu^{2} \omega^{2}+24 \mu^{3} \omega+3 \mu^{4}\right)}{4(2 \omega+\mu)^{4}}, \\
c_{2,0}^{3}=c_{0,2}^{3}=-\frac{\mu\left(39 \omega^{3}-3 \mu \omega^{2}+15 \mu^{2} \omega+3 \mu^{3}\right)}{2(2 \omega+\mu)^{3}}, \quad c_{4,0}^{3}=c_{0,4}^{3}=\frac{\mu^{2}(5 \omega+\mu)^{2}(\omega-\mu)^{2}}{8(2 \omega+\mu)^{4}} \\
\text { and } c_{3,1}^{3}=c_{1,3}^{3}=\frac{\mu^{2}(\omega-\mu)\left(65 \omega^{3}+33 \mu \omega^{2}+9 \mu^{2} \omega+\mu^{3}\right)}{2(2 \omega+\mu)^{4}} .
\end{gathered}
$$

Except for the difference in the normalization and notation conventions, these coefficients coincide with the ones found in [26].

Keeping all this in mind and determining the normalization constant $k_{N}$ by imposing that $\int \rho_{1}(r ; r) d r=1$, one finally has the expression

$$
\rho_{1}\left(r ; r^{\prime}\right)=\frac{1}{\sqrt{\pi}} \frac{1}{\sqrt{N}} \sqrt{\frac{\omega \mu}{(N-1) \omega+\mu}} e^{-a_{N}\left(r^{2}+r^{\prime 2}\right)+2 c_{N} r r^{\prime}} \sum_{t=0}^{N-1} \sum_{s=0}^{2 r} c_{t, s}^{N} r^{2 t-s} r^{\prime s}
$$

for the one-body density of the one-dimensional spinless N-fermion harmonium.

Let us now quantify the entanglement of the system by means of the linear entropy associated to the one-body density [45]:

$$
S_{L}=1-N \operatorname{Tr}\left[\rho_{1}^{2}\right]
$$

where $\Pi_{N}=N \operatorname{Tr}\left[\rho_{1}^{2}\right]$ is the purity of the system. This entanglement measure, which is a non-negative quantity that vanishes if and only if the state has Slater rank 1 and it is therefore separable, has been recently used in various two-fermion systems [13,24] as well as for various helium-like systems $[14,16,17]$ in both ground and excited states. Let us also 
point out that the linear entropy is a linearization of the von Neumann entropy, and gives a lower bound for this logarithmic entropy. Moreover, in our systems it turns out that

$$
\operatorname{Tr}\left[\rho_{1}^{2}\right]=\int \rho_{1}\left(r ; r^{\prime}\right) \rho_{1}\left(r^{\prime} ; r\right) d r^{\prime} d r=k_{N}^{2} \sum_{t, e=0}^{N-1} \sum_{s=0}^{2 t} \sum_{f=0}^{2 e} c_{t, s}^{N} c_{e, f}^{N} \mathcal{I}_{(2 t-s+f, 2 e-f+s)},
$$

where, as shown in Appendix B,

$$
\begin{aligned}
\mathcal{I}_{(n, m)}= & \mathcal{I}_{(m, n)} \\
= & \int_{-\infty}^{\infty} r^{n} r^{\prime m} e^{-2 a_{N}\left(r^{2}+r^{\prime 2}\right)+4 C_{N} r r^{\prime}} d x d y \\
= & 2 \sum_{i=0}^{n} \sum_{j=0}^{m}\left(\begin{array}{c}
n \\
i
\end{array}\right)\left(\begin{array}{c}
m \\
j
\end{array}\right)(-1)^{j} \lambda_{1}^{-(n+m-i-j+1) / 2} \lambda_{2}^{-(i+j+1) / 2} \\
& \times \Gamma\left(\frac{n+m-i-j+1}{2}\right) \Gamma\left(\frac{n+m+1}{2}\right) \frac{1}{4}\left[1+(-1)^{n+m-i-j}\right]\left[1+(-1)^{i+j}\right],
\end{aligned}
$$

with $\lambda_{1}=4\left(a_{N}-c_{N}\right)$ and $\lambda_{2}=4\left(a_{N}+c_{N}\right)$.
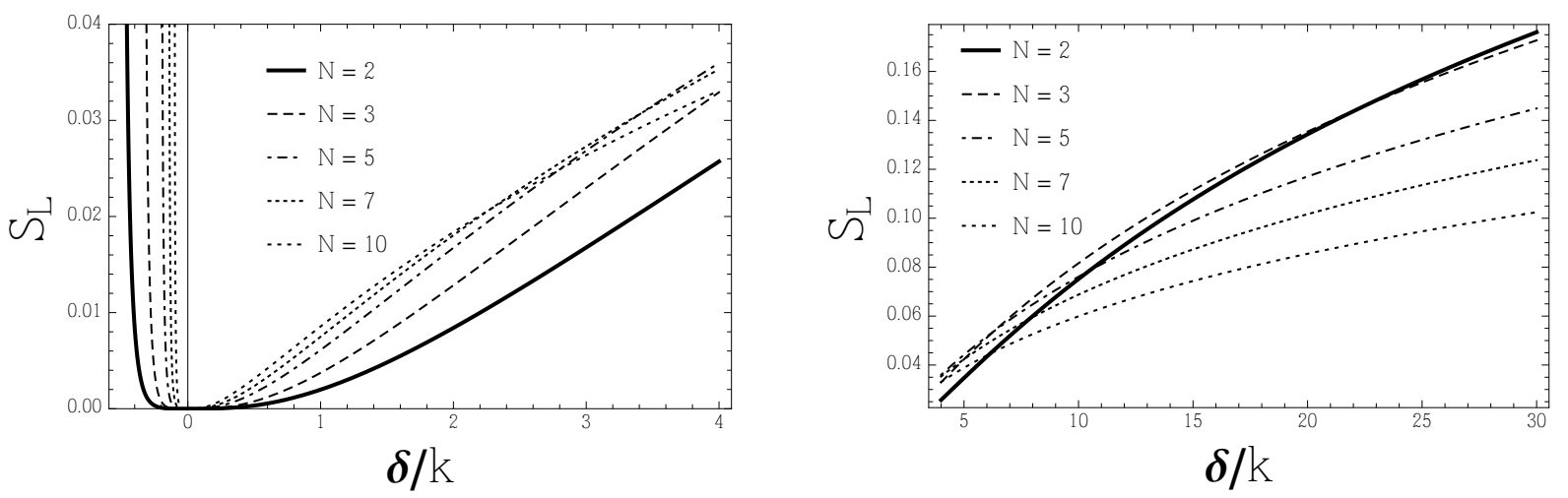

Figure 5: Linear entropy of the one-particle density matrix of the $\mathrm{N}$-fermion harmonium as a function of the coupling constant for five different values of $N$. Note the change of behavior when the strength grows.

Using expressions (40), (41) and (42), we have investigated the dependence of the linear entropy of the system on the relative interaction strength $\delta / k$, as well as on the energy. The results are given in Figure 5. A first basic observation is that for fixed $N$ the entanglement decreases (increases) when the relative interaction strength is increasing in the negative (positive) region. Moreover, for negative values of the coupling constant, the spatial entanglement of the $\mathrm{N}$-fermion harmonium grows when $N$ is increasing. For small positive values of the coupling constant (i.e., when the fermions attract each other); herein we find that for very small values of $\delta / k$ the entanglement grows again with increasing $N$. In general, we observe that for positive values of the strength we have various regimes of dependence on $N$. In the strong-coupling regime, the linear entropy grows as the number of particles is 
increasing since the purity decreases with increasing number of particles. For instance, for $\delta / k=10$ the purity is 0.92 for $N=2$ and 0.94 for $N=10$, whereas for $\delta / k=1$ is 0.998 and 0.992 respectively.

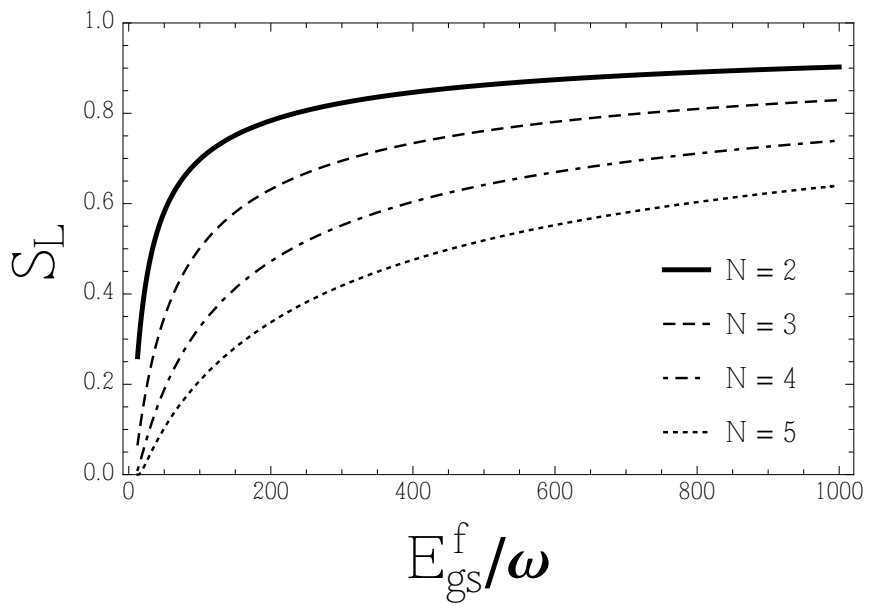

Figure 6: Linear entropy $S_{L}$ of the $\mathrm{N}$-fermion harmonium versus the ground-state energy $E_{\mathrm{gs}}^{f} / \omega$ for different values of $N$. The relation between the relative strength $\delta / k$ and the energy is stated in (43). The entanglement grows when the energy increases.

Figure 6 displays the dependence of the linear entropy of the system on the ground state energy $E_{\mathrm{gs}}^{f} / \omega$ for various values of the number of fermions $N$. We should keep in mind that the relation between the relative interaction strength and the energy is given by

$$
\delta / k=\left[\frac{2 E_{\mathrm{gs}}^{f} / \omega-1}{N^{2}-1}\right]^{2}-1 .
$$

We find that the entanglement grows when the energy increases. For $N=2$ this behavior has been recently observed not only in the Moshinsky or 2-fermion harmonium atom but also in other 2-fermion systems [13-17]. The novelty is that this behavior appears to be true also for heavier N-fermion harmonium atoms.

It is worth noting that our results can be considered as a generalization of previous results for the entanglement entropy of the fractional quantum Hall effect based in an exact matrix-product representation of the Laughlin wave function [46-48]. This well-known wave function, formulated by R. Laughlin to explain the fractional quantum Hall effect, consists of a lone Slater determinant and reads as in (27) except that there is no coupling between the electrons, that is, $\omega=\mu$ or $\delta=0$, and the domain is the complex space.

\section{Total entanglement of the spinned N-fermion harmonium}

So far, we have considered only spinless particles. This means that in the two previous sections we have determined the spatial entanglement of the N-boson and N-fermion harmonia. 
Nevertheless, our conclusions for the bosonic system also apply to the total entanglement from a qualitative point of view, because the spin part of the ground-state eigenfunction fully factorizes. For fermionic systems this is no longer true, at least when they are isolated. In fact, for the ground state of two fermions is the product of a two-boson state and an antisymmetric spin state. For a system of fermions with spin in presence of a strong magnetic field, the spin part of the eigenfunction factorizes and so the spatial part must be antisymmetric [26]. In general, however, for the isolated fermionic system the spatial and spin parts of the ground-state wave function are not separable, and antisymmetry must take into consideration both spin and position coordinates.

Let us now calculate the total entanglement of the N-fermion harmonium (i.e., the entanglement of both spatial and spin degrees of freedom) using the linear entropy as measure of entanglement. For simplicity, we consider one-dimensional models with an even number (say $2 N$ ) of spinned electrons from now on. The total spin of the system is zero, and all the spatial orbitals are doubly occupied (restricted configuration). This will eventually allow for an easy generalization to closed-shell three-dimensional systems. We define the set of spin natural orbitals in the following way:

$$
\varphi_{j}(x)=\left\{\begin{array}{cl}
\phi_{\frac{j-1}{2}}(r) \uparrow, & \text { if } j \text { is odd } \\
\phi_{\frac{j}{2}}(r) \downarrow, & \text { if } j \text { is even. }
\end{array}\right.
$$

The symbols $\uparrow$ and $\downarrow$ mean spin up and spin down, respectively, and we denote $x:=(r, \varsigma)$, $\varsigma$ being the spin coordinate.

Let us begin with the consideration of the simpler non-interacting case; that is, when $\delta=0$, and hence $\mu=\omega$. The corresponding ground-state eigenfunction is then given by the expression

$$
\begin{aligned}
\Psi^{f}\left(x_{1}, \ldots, x_{N}\right) & =\frac{1}{\sqrt{2 N !}} \sum_{J \in S_{2 N}}(-)^{J}\left[\prod_{i=1}^{2 N} \varphi_{i-1}\left(x_{J(i)}\right)\right] \\
& =\frac{1}{\sqrt{2 N !}} \sum_{J \in S_{2 N}}(-)^{J} J\left[\prod_{i=1}^{N} \uparrow_{2 i-1} \downarrow_{2 i} \phi_{i-1}^{\omega}\left(r_{2 i-1}\right) \phi_{i-1}^{\omega}\left(r_{2 i}\right)\right] .
\end{aligned}
$$

In the non-interacting case, the basis of the one-particle Hilbert space is the set of Hermite functions whose degree is less than $N$. Moreover, let us define

$$
\begin{aligned}
& H=\left\{j \in S_{2 N}: j \text { fixes } 1, \ldots, N\right\} \simeq S_{N} \\
& K=\left\{j \in S_{2 N}: j \text { fixes } N+1, \ldots, 2 N\right\} \simeq S_{N}
\end{aligned}
$$

and $\tilde{S}_{N}=H \times K$ the direct product of these two subgroups. The set $S_{2 N}^{\prime}=S_{2 N} / \tilde{S}_{N}$ is the set of right cosets in $S_{2 N}$, giving the following equivalence relation: $J \sim J^{\prime}$ if and only if there exists $\left(j, j^{\prime}\right) \in \tilde{S}_{N}$ such that $J^{\prime}=\left(j, j^{\prime}\right) J$. 
Therefore we can reorganize the expression (44) in the following form:

$$
\begin{aligned}
\Psi^{f}\left(x_{1}, \ldots, x_{N}\right) & =\frac{1}{\sqrt{2 N !}} \sum(-)^{J} \prod_{i=1}^{N} \uparrow_{J(i)} \prod_{i=N+1}^{2 N} \downarrow_{J(i)} \\
& \times\left|\begin{array}{ccc}
\phi_{0}^{\omega}\left(r_{J(1)}\right) & \cdots & \phi_{0}^{\omega}\left(r_{J(N)}\right) \\
\vdots & \ddots & \vdots \\
\phi_{N}^{\omega}\left(r_{J(1)}\right) & \cdots & \phi_{N}^{\omega}\left(r_{J(N)}\right)
\end{array}\right|\left|\begin{array}{ccc}
\phi_{0}^{\omega}\left(r_{J(N+1)}\right) & \cdots & \phi_{0}^{\omega}\left(r_{J(2 N)}\right) \\
\vdots & \ddots & \vdots \\
\phi_{N}^{\omega}\left(r_{J(N+1)}\right) & \cdots & \phi_{N}^{\omega}\left(r_{J(2 N)}\right),
\end{array}\right|
\end{aligned}
$$

where the sum runs over a representative of each coset. The choice of the representative $J \in[J] \in S_{2 N}^{\prime}$ is immaterial. The number of summands is

$$
\left|S_{2 N} / \tilde{S}_{N}\right|=\frac{\left|S_{2 N}\right|}{\left|\tilde{S}_{N}\right|}=\frac{(2 N) !}{(N !)^{2}}=\left(\begin{array}{c}
2 N \\
N
\end{array}\right) .
$$

For instance, for the four-harmonium $(N=4)$, the eigenfunction can be written in the alternative form:

$$
\begin{aligned}
\Psi^{f}\left(x_{1}, x_{2}, x_{3}, x_{4}\right) & =\frac{\omega^{2}}{\pi} \frac{1}{\sqrt{3 !}} e^{-\frac{\omega}{2}\left(r_{1}^{2}+r_{2}^{2}+r_{3}^{2}+r_{4}^{2}\right)}\left[\left(\uparrow_{1} \uparrow_{2} \downarrow_{3} \downarrow_{4}+\downarrow_{1} \downarrow_{2} \uparrow_{3} \uparrow_{4}\right) \mathcal{V}_{(1,2)} \mathcal{V}_{(3,4)}\right. \\
& \left.-\left(\uparrow_{1} \uparrow_{3} \downarrow_{2} \downarrow_{4}+\downarrow_{1} \downarrow_{3} \uparrow_{2} \uparrow_{4}\right) \mathcal{V}_{(1,3)} \mathcal{V}_{(2,4)}+\left(\uparrow_{1} \uparrow_{4} \downarrow_{2} \downarrow_{3}+\downarrow_{1} \downarrow_{4} \uparrow_{2} \uparrow_{3}\right) \mathcal{V}_{(1,4)} \mathcal{V}_{(2,3)}\right]
\end{aligned}
$$

The one-body reduced density matrix corresponding to the wave function (45) is diagonal in spin space and is given by the expression

$$
\rho_{1}\left(x, x^{\prime}\right)=\left(\begin{array}{cc}
\rho_{1}^{\uparrow \uparrow}\left(r, r^{\prime}\right) & 0 \\
0 & \rho_{1}^{\downarrow \downarrow}\left(r, r^{\prime}\right)
\end{array}\right) .
$$

Moreover, it is clear that

$$
\begin{aligned}
\rho_{1}^{\uparrow \uparrow}\left(r, r^{\prime}\right) & =\rho_{1}^{\downarrow \downarrow}\left(r, r^{\prime}\right)=\left.\int d x_{2} \cdots d x_{2 N} \Psi^{f}\left(r, \varsigma_{1}, x_{2}, \ldots, x_{N}\right) \Psi^{f}\left(r^{\prime}, \varsigma_{1}, x_{2}, \ldots, x_{N}\right)\right|_{\varsigma_{1}=\uparrow} \\
& =\frac{1}{2 N} \frac{1}{(N-1) !} \int d r_{2} \cdots d r_{N}\left|\begin{array}{ccc}
\varphi_{0}(r) & \cdots & \varphi_{0}\left(r_{N}\right) \\
\vdots & \ddots & \vdots \\
\varphi_{N}(r) & \cdots & \varphi_{N}\left(r_{N}\right)
\end{array}\right|\left|\begin{array}{ccc}
\varphi_{0}\left(r^{\prime}\right) & \cdots & \varphi_{0}\left(r_{N}\right) \\
\vdots & \ddots & \vdots \\
\varphi_{N}\left(r^{\prime}\right) & \cdots & \varphi_{N}\left(r_{N}\right)
\end{array}\right| \\
& =\frac{1}{2 N} \sum_{i=0}^{N-1} \varphi_{i}(r) \varphi_{i}\left(r^{\prime}\right) .
\end{aligned}
$$

The occupation numbers appear twice, what is a well-known result for atomic and molecular scientists.

Let us now consider the general interacting case, for which $\delta \neq 0$, and hence $\mu \neq \omega$. The ground-state eigenfunction for the Hamiltonian (1) is similar to (22), except that each spatial orbital is doubly occupied. For the same reasons as in the spinless case, the collective mode 
$\xi_{2 N}$ occupies the Hermite function of degree zero. The other coordinates $\left\{\xi_{m}\right\}_{m=1}^{2 N-1}$ occupy the other spatial orbitals in such a way that the total wave function is totally antisymmetric under interchange the coordinates $\left\{x_{m}\right\}_{m=1}^{2 N}$. The ground-state eigenfunction schematically reads

$$
\Psi^{f}\left(x_{1}, \ldots, x_{2 N}\right)=\frac{1}{\sqrt{2 N !}} \sum_{J \in S_{2 N}}(-)^{J}\left[\prod_{i=1}^{N} \uparrow_{J(i)} \prod_{i=N+1}^{2 N} \downarrow_{J(i)}\right] J\left[\phi_{0}^{\omega}\left(\xi_{2 N}\right) \phi_{0}^{\mu}(\cdot) \prod_{m=1}^{N} \phi_{m}^{\mu}(\cdot) \phi_{m}^{\mu}(\cdot)\right] .
$$

For instance, in the particular case $N=4$ the eigenfunction has the form

$$
\begin{aligned}
\Psi^{f}\left(x_{1}, x_{2}, x_{3}, x_{4}\right) & =\frac{1}{\sqrt{4 !}} \sum_{J \in S_{4}}(-)^{J} \uparrow_{J(1)} \uparrow_{J(2)} \downarrow_{J(3)} \downarrow_{J(4)} J\left[\phi_{0}^{\omega}\left(\xi_{4}\right) \phi_{1}^{\mu}\left(\xi_{1}\right) \phi_{0}^{\mu}\left(\xi_{3}\right) \phi_{1}^{\mu}\left(\xi_{2}\right)\right] \\
& =(\text { const. }) \sum_{J \in S_{4}}(-)^{J} \uparrow_{J(1)} \uparrow_{J(2)} \downarrow_{J(3)} \downarrow_{J(4)} J\left[\xi_{1} \xi_{2}\right] e^{-\frac{\omega}{2} \xi_{4}-\frac{\mu}{2}\left(\xi_{1}^{2}+\xi_{2}^{2}+\xi_{3}^{2}\right)} \\
& =\text { (const.) } \sum_{S_{4}^{\prime}}(-)^{J} \uparrow_{J(1)} \uparrow_{J(2)} \downarrow_{J(3)} \downarrow_{J(4)} \mathcal{V}_{(J(1), J(2))} \mathcal{V}_{(J(3), J(4))} e^{-\frac{\omega}{2} \xi_{4}-\frac{\mu}{2}\left(\xi_{1}^{2}+\xi_{2}^{2}+\xi_{3}^{2}\right)} .
\end{aligned}
$$

Using again that an antisymmetric polynomial is equal to a symmetric polynomial multiplied by a Vandermonde determinant [43], we can write the ground-state eigenfunction of the general interacting spinned $\mathrm{N}$-fermion system as:

$$
\begin{aligned}
\Psi^{f}\left(x_{1}, \ldots, x_{2 N}\right)= & (\text { const. }) e^{-\frac{\omega-\mu}{2} \xi_{2 N}^{2}-\frac{\mu}{2} \sum_{i=1}^{2 N} r_{i}^{2}} \\
& \times \sum_{S_{2 N}^{\prime}}(-)^{J}\left[\prod_{i=1}^{N} \uparrow_{J(i)} \prod_{i=N+1}^{2 N} \downarrow_{J(i)}\right] \mathcal{V}_{(J(1), \ldots, J(N))} \mathcal{V}_{(J(N+1), \ldots, J(2 N))} .
\end{aligned}
$$

and the corresponding energy is $E_{\mathrm{gs}}^{f}=\frac{1}{2}(\omega+\mu)+\mu\left(N^{2}-1\right)$. Let us define $\eta=r+r_{2}+\cdots+r_{N}$ and $\eta^{\prime}=r^{\prime}+r_{2}+\cdots+r_{N}$. To compute the one-density we use twice the Hubbard-Stratonovich identity. First to compute $N$ integrals with $\zeta^{\prime}=r_{N+1}+\cdots+r_{2 N}$, and second to compute $N-1$ integrals with $\zeta=r_{2}+\cdots+r_{N}$. Each diagonal element of the one-body density matrix reads:

$$
\begin{aligned}
& \rho_{1}^{\uparrow \uparrow}\left(r, r^{\prime}\right)=\rho_{1}^{\downarrow \downarrow}\left(r, r^{\prime}\right) \\
& =(\text { const. }) e^{-a\left(r^{2}+r^{\prime 2}\right)} \int d r_{2} \cdots d r_{N} e^{-2 a\left(r_{2}^{2}+\cdots+r_{N}^{2}\right)+b_{2 N}\left(\eta^{2}+\eta^{\prime 2}\right)} \int d z e^{-2 b_{2 N} z^{2}} e^{N \frac{b_{2 N}^{2}}{2 a}\left(\eta+\eta^{\prime}+2 z\right)^{2}} \\
& \quad \times \mathcal{V}_{(r,, 2, \ldots, N)} \mathcal{V}_{\left(r^{\prime}, 2, \ldots, N\right)} \int d z_{N+1} \cdots d z_{2 N} \mathcal{V}_{\left(z_{N+1}, \ldots, z_{2 N}\right)}^{2} \prod_{j=N+1}^{2 N} e^{-z_{j}^{2}}
\end{aligned}
$$

where $z_{j}:=\sqrt{2 a}\left[r_{j}-\frac{b_{2 N}}{2 a}\left(\eta+\eta^{\prime}+2 z\right)\right]$. Once again, the expression on the right is the total integral of the product of two Slater determinants, that is,

$$
\int d z_{N+1} \cdots d z_{2 N} \mathcal{V}_{\left(z_{N+1}, \ldots, z_{2 N}\right)}^{2} \prod_{j=N+1}^{2 N} e^{-z_{j}^{2}}
$$


is a real constant. Therefore

$$
\begin{aligned}
& \rho_{1}^{\uparrow \uparrow}\left(r, r^{\prime}\right)=\rho_{1}^{\downarrow \downarrow}\left(r, r^{\prime}\right) \\
& =(\text { const. }) e^{-a\left(r^{2}+r^{\prime 2}\right)} \int d r_{2} \cdots d r_{N} e^{-2 a\left(r_{2}^{2}+\cdots+r_{N}^{2}\right)+b_{2 N}\left(\eta^{2}+\eta^{\prime 2}\right)+d_{N}\left(\eta+\eta^{\prime}\right)^{2}} \mathcal{V}_{r, 2, \ldots, N} \mathcal{V}_{r^{\prime}, 2, \ldots, N} \\
& =\text { (const.) } e^{-a\left(r^{2}+r^{\prime 2}\right)+b_{2 N}\left(r^{2}+r^{\prime 2}\right)+d_{N}\left(r+r^{\prime}\right)^{2}} \int d z e^{-2 g_{N} z^{2}} e^{(N-1) \frac{g_{N}^{2}}{2 a}\left(r+r^{\prime}+2 z\right)^{2}} \\
& \quad \times \int d z_{2} \cdots d z_{N} \mathcal{V}_{z_{r}, z_{2}, \ldots, z_{N}} \mathcal{V}_{z_{r}^{\prime}, z_{2}, \ldots, z_{N}} \prod_{j=2}^{N} e^{-z_{j}^{\prime 2}},
\end{aligned}
$$

where $z_{j}^{\prime}:=\sqrt{2 a}\left[r_{j}-\frac{g_{N}}{2 a}\left(r+r^{\prime}+2 z\right)\right], d_{N}=\frac{1}{8 N} \frac{(\mu-\omega)^{2}}{\mu+\omega}$ and $g_{N}=\frac{\mu-\omega}{2 N} \frac{\mu}{\mu+\omega}$. As in the spinless case, let us define

$$
\tilde{q}_{\left(r, r^{\prime}\right)}=\sqrt{\mu}\left[r-\frac{1}{2} \beta_{2 N}^{2}\left(r+r^{\prime}\right)\right] \quad \text { and } \quad \tilde{c}_{2 N}=\frac{(\mu-\omega)^{2}}{(2 N-1) \omega+\mu} \frac{2 N-1}{8 N}
$$

with $\beta_{N}$ as defined in (33). Then

$$
\begin{aligned}
\rho_{1}^{\uparrow \uparrow}\left(r, r^{\prime}\right) & =\rho_{1}^{\downarrow \downarrow}\left(r, r^{\prime}\right)=\frac{1}{2 \pi} \frac{1}{\sqrt{N}} \sqrt{\frac{2 \omega \mu}{(2 N-1) \omega+\mu}} \\
& \times e^{-a_{N}^{\prime}\left(r^{2}+r^{\prime 2}\right)+2 \tilde{c}_{2 N} r r^{\prime}} \int d u e^{-u^{2}} \sum_{j=0}^{N-1} \frac{1}{2^{j} j !} H_{j}\left[\tilde{q}_{\left(r, r^{\prime}\right)}-\beta_{2 N} u\right] H_{j}\left[\tilde{q}_{\left(r^{\prime}, r\right)}-\beta_{2 N} u\right],
\end{aligned}
$$

with $a_{N}^{\prime}:=a-b_{2 N}-\tilde{c}_{2 N}$.

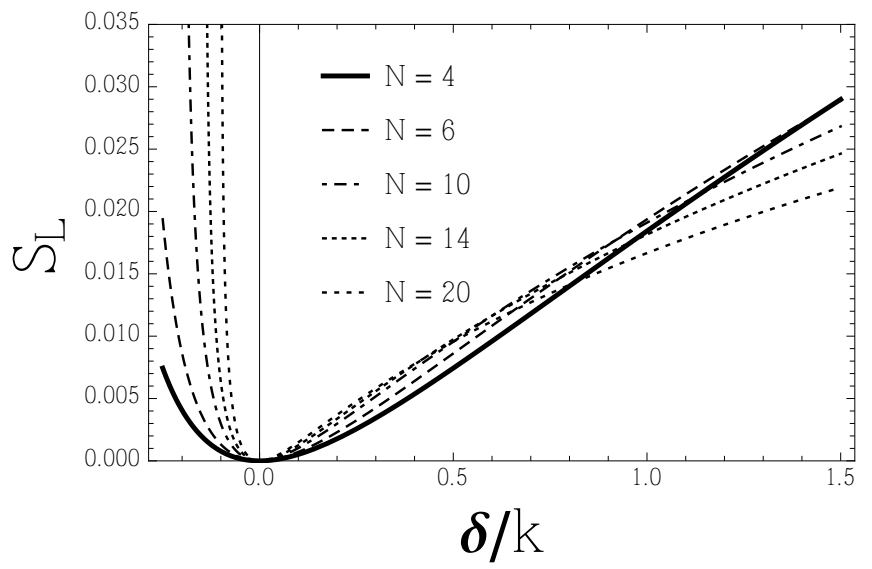

Figure 7: Linear entropy of the spinned N-fermion harmonium as a function of the relative coupling constant for various values of $N$. Qualitatively, it is similar to the spinless case in Fig. 5. 
The expression (53) is similar to (34), except that we have an even number of fermions: $N \rightarrow 2 N$ and $c_{N} \rightarrow \tilde{c}_{2 N}$. It is now clear that the conclusions for the spinless fermionic case also hold for the full spin case from a qualitative point of view. Figure 7 plots the linear entropy for the spinless and the spinned cases as functions of the relative strength.

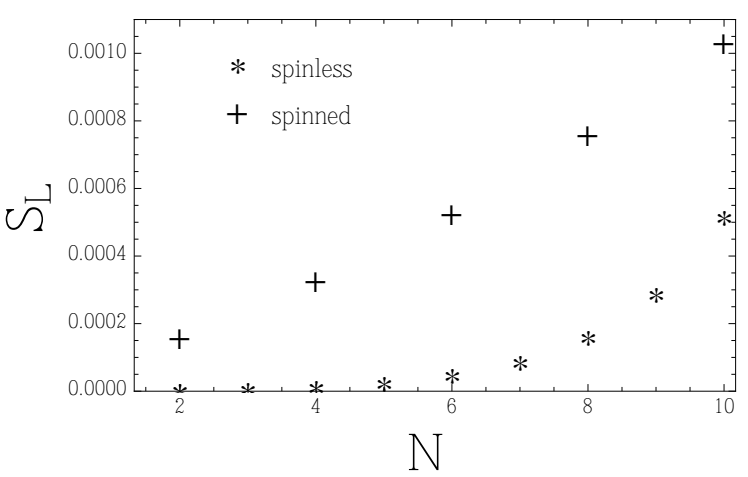

(a) $\delta / k=-1 / 15$

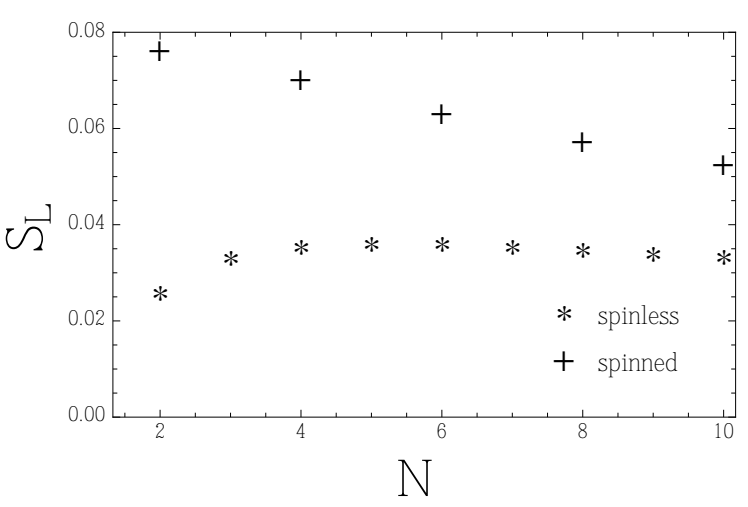

(c) $\delta / k=4$

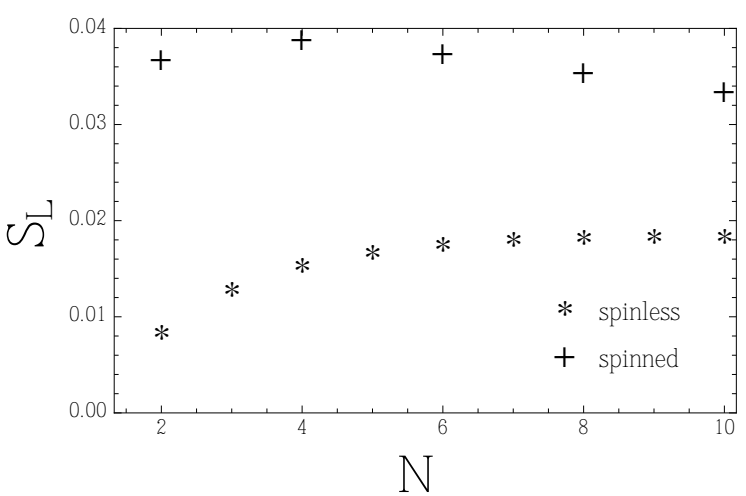

(b) $\delta / k=2$

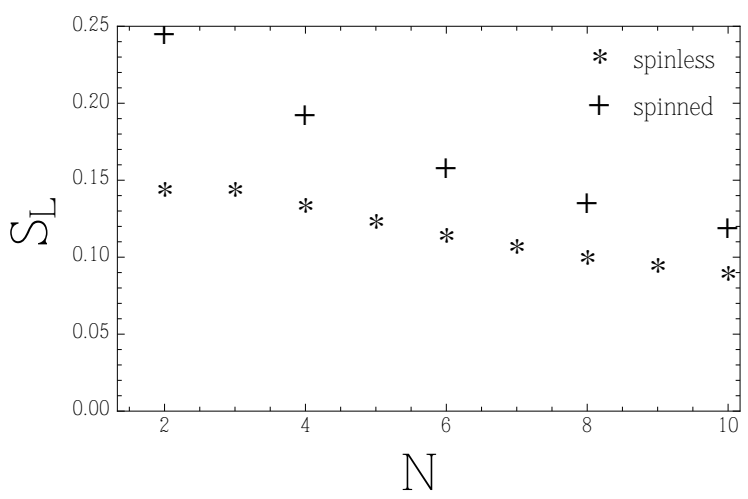

(d) $\delta / k=22$

Figure 8: Linear entropy of the one-body reduced density matrix for the spinless and spinned N-fermion harmonium as a function of the number of particles for different values of $\delta / k$.

For completeness, we have numerically investigated the dependence of the linear entropy on the number of particles for some specific values of the relative coupling in both spinless and spinned cases (i.e., for spatial and total entanglements). Figure 8 exhibits the results for $\delta / k \in\{-1 / 15,2,4,22\}$. In the spinned case the behavior is similar to the spinless system, so that the total entanglement behaves similarly as the spatial entanglement. In fact, for small values of the coupling the entropy grows when increasing the number of particles, while in the strong-coupling regime the situation is the opposite. The inclusion of the spin tends to enhance the entropy of the system, being most important this phenomenon for small values of the coupling constant. Note that in turn the contributions of the spin and the spatial one are of comparable size.

Finally, let us investigate the relative behavior of entanglement and energy. This is done 


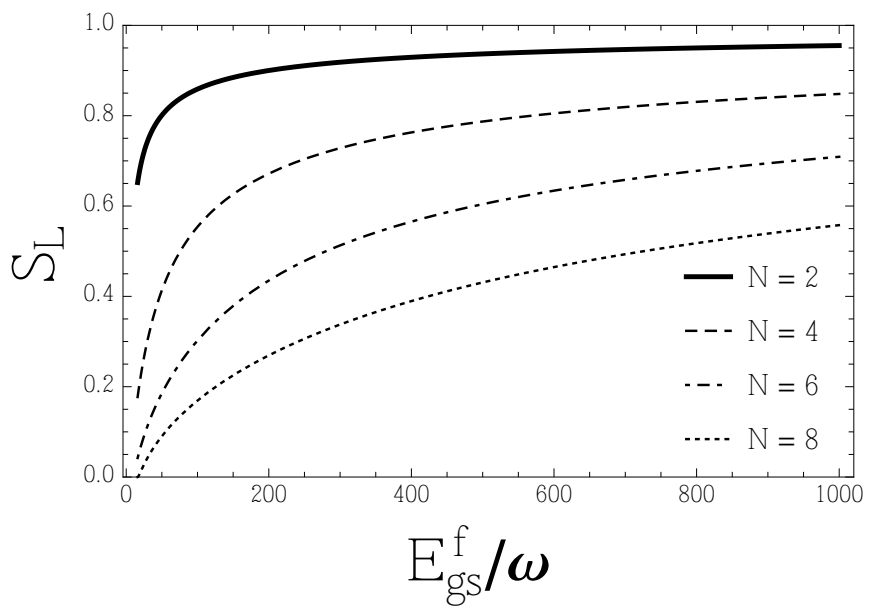

Figure 9: Linear entropy $S_{L}$ of the N-fermion spinned harmonium versus the ground-state energy $E_{\mathrm{gs}}^{f} / \omega$ for different values of $N$. The relation between the relative strength $\delta / k$ and the energy is stated in (54).

in Figure 9, where the linear entropy as a function of the ground-state energy is shown for some values of the number of particles. Let us recall here that the relation between the relative coupling and the energy is given by

$$
\delta / k=\left[\frac{2 E_{\mathrm{gs}}^{f} / \omega-1}{2 N^{2}-1}\right]^{2}-1 .
$$

As in the spinless case, the entropy grows when increasing the ground-state energy. For very large values of the dimensionless energy $E_{\mathrm{gs}}^{f} / \omega$ (i.e., very large values of the relative strength $\delta / k)$ the gap between the spinless and spinned cases decreases.

\section{Concluding remarks}

In this work we have shown that some entanglement features of finite many-particle systems can be understood to a certain extent by purely kinematical considerations. This is done by explicitly analyzing the entanglement of the N-boson and N-fermion harmonium systems. This has been possible because for these harmonic systems we have been able to calculate analytically not only the one-body reduced matrix for bosons and fermions, but also the von Neumann entropy in the bosonic case as well as the linear entropy in the fermionic case. In doing so, we complement and extend to harmonic systems with an arbitrary number of particles the study of entanglement recently done for various two-electron models [13,21-25] as well as some helium-like systems $[6,14,15,17]$ and certain quantum complex networks [49].

We have determined the entanglement of these harmonic systems for both spatial and spin degrees of freedom analytically in terms of the number of particles and the relative 
interaction strength (or coupling constant). We have used the von Neumann entropy and the linear entropy as entanglement quantifiers in the bosonic and fermionic systems, respectively. We have found that for positive couplings the entanglement of the 3-boson harmonium atom is bigger than the one of the 2-boson system, but in general the entanglement of the N-boson harmonium decreases when $N$ is increasing. Moreover, the entanglement of a given N-boson system grows when the positive coupling constant is increasing; that is, when the positive value if the strength of interparticle interaction relative to the confinement well is increasing. On the other hand, globally speaking the spatial entanglement of the N-fermion harmonium grows when $N$ is increasing for both negative and sufficiently small positive values of the coupling constant. Moreover, the entanglement behavior is opposite in the positive strongcoupling regime. On the other hand, entanglement of a given N-fermion system grows when the coupling constant is increasing. The contribution of the spin degree of freedom to the entanglement of the $\mathrm{N}$-fermion system is shown to be of positive comparable size to the contribution of the spatial degrees of freedom.

Summarizing, we have shown that in the repulsive case and in the attractive case for relatively small values of the coupling constant, the entanglement of both bosonic and fermionic N-harmonium atoms grows when the number of particles is increasing, basically because purity decreases. However, in the regime of strong coupling the situation is exactly the opposite: purity increases (and hence entanglement decreases) by adding particles to the system.

\section{Acknowledgments}

CLBR was supported by a Francisco José de Caldas scholarship, funded by Colciencias. He very much appreciates the warm atmosphere of the Departamento de Física Atómica Molecular y Nuclear, at the Universidad de Granada. IVT and JSD gratefully acknowledge the MINECO grant FIS2011-24540 and the excellence grant FQM-7276 of the Junta de Andalucía. In addition, the authors are most grateful to A. R. Plastino as well as to J. L. Alonso, A. Botero, J. M. Gracia-Bondía and J. C. Várilly for helpful and illuminating discussions.

\section{A Calculation of the integral $\int_{-\infty}^{\infty} e^{-u^{2}} H_{k}(a-c u) H_{k}(b-c u) d u$}

In this section we calculate the integral

$$
\int_{-\infty}^{\infty} e^{-u^{2}} H_{k}(a-c u) H_{k}(b-c u) d u
$$


where $a, b$ and $c$ are real numbers and $H_{k}(x)$ is the Hermite polynomial of degree $k$. We use the following property of the Hermite polynomials

$$
H_{n}(-z)=(-1)^{n} H_{n}(z)
$$

as well as the power series of these polynomials around $z=z_{0}$

$$
H_{n}(z)=\sum_{k=0}^{n} 2^{k}\left(\begin{array}{l}
n \\
k
\end{array}\right) H_{n-k}\left(z_{0}\right)\left(z-z_{0}\right)^{k}
$$

This expression allows us to rewrite each Hermite polynomial in the following form:

$$
H_{k}(a-c u)_{z_{0}=-a}^{=}(-1)^{k} \sum_{n_{1}=0}^{k}(2 c)^{n_{1}}\left(\begin{array}{c}
k \\
n_{1}
\end{array}\right) H_{k-n_{1}}(-a) u^{n_{1}} .
$$

Inserting (56) into (55) we obtain

$$
\begin{aligned}
& \int_{-\infty}^{\infty} e^{-u^{2}} H_{k}(a-c u) H_{k}(b-c u) d u \\
& =\int_{-\infty}^{\infty} e^{-u^{2}}(-1)^{k} \sum_{n_{1}=0}^{k}(2 c)^{n_{1}}\left(\begin{array}{c}
k \\
n_{1}
\end{array}\right) H_{k-n_{1}}(-a) u^{n_{1}}(-1)^{k} \sum_{n_{2}=0}^{k}(2 c)^{n_{2}}\left(\begin{array}{c}
k \\
n_{2}
\end{array}\right) H_{k-n_{2}}(-b) u^{n_{2}} \\
& \quad=\sum_{\substack{n_{1}, n_{2}=0 \\
n_{1}+n_{2} \text { even }}}^{k}(2 c)^{n_{1}+n_{2}}\left(\begin{array}{c}
k \\
n_{1}
\end{array}\right)\left(\begin{array}{c}
k \\
n_{2}
\end{array}\right) H_{k-n_{1}}(-a) H_{k-n_{2}}(-b) \Gamma\left(\frac{n_{1}+n_{2}+1}{2}\right)
\end{aligned}
$$

\section{B The Gaussian integral $\mathcal{I}_{(n, m)}$}

In the following, we calculate the integral

$$
\mathcal{I}_{(n, m)}=\int_{-\infty}^{\infty} \int_{-\infty}^{\infty} x^{n} y^{m} e^{-a\left(x^{2}+y^{2}\right)+2 c x y} d x d y
$$

Here we perform the change of coordinates

$$
x=u+v \quad \text { and } \quad y=u-v
$$

and the integral now reads:

$$
\mathcal{I}_{(n, m)}=2 \sum_{i=0}^{n} \sum_{j=0}^{m}\left(\begin{array}{c}
n \\
i
\end{array}\right)\left(\begin{array}{c}
m \\
j
\end{array}\right)(-1)^{j} \int_{-\infty}^{\infty} \int_{-\infty}^{\infty} u^{n+m-i-j} v^{i+j} e^{-\lambda_{1} u^{2}-\lambda_{2} v^{2}} d u d v
$$

with $\lambda_{1}=2(a-c)$ and $\lambda_{2}=2(a+c)$. From the form of the (double) integral, on using polar coordinates in the $(u, v)$-plane, $u=R \cos \phi$ and $v=R \sin \phi$, since the exponential is an even 
function of $\phi$, the double integral vanishes if $i+j$ is odd. Switching $u$ and $v$ in the polar coordinates we likewise see that it vanishes if $(m+n)-(i+j)$ is odd.

The integral thus becomes

$$
\begin{aligned}
& \mathcal{I}_{(n, m)} \\
& =2 \sum_{i=0}^{n} \sum_{j=0}^{m}\left(\begin{array}{c}
n \\
i
\end{array}\right)\left(\begin{array}{c}
m \\
j
\end{array}\right)(-1)^{j} \lambda_{1}^{-(n+m-i-j+1) / 2} \lambda_{2}^{-(i+j+1) / 2} \int_{-\infty}^{\infty} \int_{-\infty}^{\infty} u^{n+m-i-j} v^{i+j} e^{-u^{2}-v^{2}} d u d v \\
& =2 \sum_{i=0}^{n} \sum_{j=0}^{m}\left(\begin{array}{c}
n \\
i
\end{array}\right)\left(\begin{array}{c}
m \\
j
\end{array}\right)(-1)^{j} \lambda_{1}^{-(n+m-i-j+1) / 2} \lambda_{2}^{-(i+j+1) / 2} \\
& \quad \times \Gamma\left(\frac{n+m-i-j+1}{2}\right) \Gamma\left(\frac{n+m+1}{2}\right) \frac{1}{4}\left[1+(-1)^{n+m-i-j}\right]\left[1+(-1)^{i+j}\right] .
\end{aligned}
$$

\section{References}

[1] A. Szabo and N. Ostlund, Modern Quantum Chemistry (Dover, New York, 1996).

[2] T. Helgaker, P. Jorgensen and J. Olsen, Molecular Electronic Structure Theory (Wiley, Chichester, 2000).

[3] M. A. Nielsen and I.L. Chuang, Quantum Computation and Quantum Information (Cambridge University Press, Cambridge, 2010).

[4] R. Horodecki, P. Horodecki. M. Horodecki and K. Horodecki Rev. Mod. Phys. 81, 865 (2009).

[5] J. Schliemann, J. I. Cirac, M. Kuś, M. Lewenstein and Daniel Loss, Phys. Rev. A 64, 022303 (2001).

[6] Z. Huang and S. Kais, Chem. Phys. Lett. 413, 1 (2005).

[7] H. Wang and S. Kais, Isr. J. Chem. 47, 59 (2007).

[8] F. Benatti, R. Floreanini and U. Marzolino, J. Phys. B: At. Mol. Opt. Phys. 44, 091001 (2010).

[9] F. Benatti, R. Floreanini and U. Marzolino, Phys. Rev. A 89, 032326 (2014).

[10] M. Tichy, F. Mintert and A. Buchleitner, J. Phys. B: At. Mol. Opt. Phys. 44, 192001 (2011).

[11] L. Amico, R. Fazio, A. Osterloh and V. Vedral, Rev. Mod. Phys. 80, 517 (2008).

[12] R. O. Esquivel, M. Molina-Espíritu, F. Salas, C. Barrientos, J. S. Dehesa and J.A. Dobado, Decoding the building bloks of life from the perspective of quantum information, In P. Bracken (editor), Advances in Quantum Mechanics, ISBN: 978- 953-51-1089-7, InTech (2013).

[13] D. Manzano, A. R. Plastino, J.S. Dehesa and T. Koga, J. Phys. B: At. Mol. Opt. Phys. 43, 275301 (2010).

[14] J. S. Dehesa, T. Koga, R. J. Yáñez, A. R. Plastino and R. O. Esquivel, J. Phys. B: At. Mol. Opt. Phys. 45, 015504 (2012); see corrigendum, ibid, 45239501 (2012). 
[15] G. Benenti, S. Siccardi and G. Strini, Eur. J. Phys. D 67, 83 (2013).

[16] T. S. Hofer, Front. Chem.: Theor. Comput. Chem. 1, 24 (2013).

[17] C. H. Lin, Y. C. Lin and Y. K. Ho, Few-Body Syst. 54, 2147 (2013).

[18] R. O. Esquivel, N. Flores-Gallegos, M. Molina-Espíritu, A. R. Plastino, J. C. Angulo, J. Antolin and J. S. Dehesa, J. Phys. B: At. Mol. Opt. Phys. 44, 175101 (2011).

[19] R. O. Esquivel, M. Molina-Espíritu, A. R. Plastino and J.S. Dehesa, Preprint submitted (2013).

[20] P. Lévay and P. Vrana, Phys. Rev. A 78, 022329 (2008).

[21] W. Heisenberg, Z. Physik 38, 411 (1926).

[22] M. Moshinsky, Am. J. Phys. 36, 52 (1968), Erratum: ibid, 36, 763 (1968).

[23] J. Pipek and I. Nagy, Phys. Rev. A 79, 052501 (2009).

[24] R. J. Yáñez, A. R. Plastino and J. S. Dehesa Eur. Phys. J. D 56, 141 (2010).

[25] C. L. Benavides-Riveros, J. M. Gracia-Bondía and J. C. Várilly, Phys. Rev. A 86, 022525 (2012).

[26] C. Schilling, Phys. Rev. A 88, 042105 (2013).

[27] J. R. Armstrong, N. T. Zinner, D. V. Fedorov and A. S. Jensen, J. Phys. B: At. Mol. Opt. Phys. 44, 055303 (2011).

[28] L. Bombelli, R. K. Koul, J. Lee and R. D. Sorkin, Phys. Rev. D 34, 373 (1986).

[29] N. F. Johnson and M. C. Payne, Phys. Rev. Lett. 67, 1157 (1991).

[30] M. Srednicki, Phys. Rev. Lett. 71, 666 (1993).

[31] J. R. Armstrong, N. T. Zinner, D. V. Fedorov and A. S. Jensen, Phys. Rev. E 85, 021117 (2012).

[32] P. Kościk and A. Okopińska, Few-Body Syst. 54, 1637 (2013).

[33] Z. Wang, A. Wang, Y. Yang and X. Li, Commum. Theor. Phys. 58, 639 (2012).

[34] C. Schilling, D. Gross and M. Christandl, Phys. Rev. Lett. 110, 040404 (2013).

[35] A. Calles and M. Moshinsky, Am. J. Phys. 38, 456 (1970).

[36] G. Gidofalvi and D. A. Mazziotti, Phys. Rev. A 69, 042511 (2004).

[37] D. A. Mazziotti, Phys. Rev. Lett. 108, 263002 (2012).

[38] O. Gritsenko, K. Pernal, and E. J. Baerends, J. Chem. Phys. 122, 204102 (2005).

[39] J. P. Dahl, Can. J. Chem. 87, 784 (2009).

[40] Ph. Blanchard, J. M. Gracia-Bondía and J. C. Várilly, Int. J. Quant. Chem. 112, 1134 (2012).

[41] R. Littlejohn, Phys. Reports 138, 193 (1986). 
[42] C. J. Pethick and L. P. Pitaevskii, Phys. Rev. A 62, 033609 (2000).

[43] C. Procesi, Lie Groups. An approach trough invariants and representations (Springer, New York, 2007).

[44] R. B. Laughlin, Phys. Rev. Lett. 50, 1395 (1983).

[45] A. R. Plastino, D. Manzano and J. S. Dehesa, Europhys. Lett. 86, 20005 (2009).

[46] M. Haque, O. Zozulya, and K. Schoutens, Phys. Rev. Lett. 98, 060401 (2007).

[47] S. Iblisdir, J. I. Latorre, and R. Orús, Phys. Rev. Lett. 98, 060402 (2007).

[48] J. I. Latorre and A. Riera, J. Phys. A: Math. Theor. 42, 504002 (2009).

[49] A. Cardillo, F. Galve, D. Zueco and J. Gómez-Gardeñes, Phys. Rev. A 87, 052312 (2013). 